Meta

Journal des tradlucteurs

Translators' Journal

\title{
La traduction en français des jeux linguistiques de Gödel, Escher, Bach
}

\section{Robert M. French et Jacqueline Henry}

Volume 33, numéro 3, septembre 1988

URI : https://id.erudit.org/iderudit/002774ar

DOI : https://doi.org/10.7202/002774ar

Aller au sommaire du numéro

Éditeur(s)

Les Presses de l'Université de Montréal

ISSN

0026-0452 (imprimé)

1492-1421 (numérique)

Découvrir la revue

Citer cet article

French, R. M. \& Henry, J. (1988). La traduction en français des jeux

linguistiques de Gödel, Escher, Bach. Meta, 33(3), 331-340.

https://doi.org/10.7202/002774ar d'utilisation que vous pouvez consulter en ligne. 


\title{
LA TRADUCTION EN FRANÇAIS DES JEUX LINGUISTIQUES DE GÖDEL, ESCHER, BACH
}

\author{
ROBERT M. FRENCH ET JACQUELINE HENRY, TRADUCTEURS \\ Ann Arbor, Michigan, U.S.A. et Paris, France
}

La grande originalité de Gödel, Escher, Bach, an Eternal Golden Braid, de Douglas R. Hofstadter, est que l'auteur s'est efforcé d'entremêler un fond scientifique très dense et un style qu'il qualifie lui-même de carrollien. Sur la jaquette du livre figure en effet la mention "A metaphorical fugue on minds and machines in the spirit of Lewis Carroll ", ce qui donne déjà une idée des difficultés, mais aussi des défis et des joies qui attendent les traducteurs d'un tel ouvrage*.

Quelques mots, avant de nous lancer plus avant dans l'analyse des divertissements linguistiques de Gödel, Escher, Bach, sur son contenu et sa structure. Le thème central de ce livre est le théorème d'incomplétude de Gödel et les liens entre les concepts contenus dans sa démonstration et la conscience, mais ses vingt chapitres " techniques " couvrent un immense champ intellectuel qui va de la logique formelle à la biologie moléculaire en passant par les fugues de Bach, les koâns zen, les tableaux d'Escher et l'informatique actuelle. Entre ces chapitres techniques viennent s'intercaler des dialogues dont les principaux personnages sont la Tortue, Achille, le Crabe et un Fourmilier. Chacun de ces dialogues, reprenant le genre de "What the Tortoise said to Achilles", texte de L. Carroll intégralement repris par Hofstadter, introduit le ou les thème(s) du chapitre suivant.

Autre point important mis en valeur par le titre des dialogues : D.R. Hofstadter fait un parallèle avec des compositions musicales de Bach. On trouve ainsi la "Sonate pour Achille seul » (Sonata for Unaccompanied Achilles), le "Canon Cancrizans " (Crab Canon), la "Kourte G'avotte " (Air on G's string), le "Canon par augmentation d'intervalles " (Canon by Intervallic Augmentation), etc. Ce parallèle ne s'arrête d'ailleurs pas aux titres des dialogues, puisque leurs personnages représentent des voix et leurs répliques contiennent un thème, des imitations, des pauses, des strettes, etc. Ainsi, on peut lire, dans la version originale annotée de Gödel, Escher, Bach que D.R. Hofstadter a soigneusement préparée à l'intention des traducteurs, les explications suivantes au sujet du dialogue Ant Fugue ("Fourmugue ") :

- Fugue theme, first voice (en marge de la première réplique d'Achille);

- Fugue theme, second voice (en marge de la première réplique du Crabe);

- Fugue theme (il s'agit de la proposition "I know the rest of you won't believe this");

- Third voice enters with theme (première réplique du Fourmilier, qui dit " $I$ know the rest of you won't believe this");

- etc.

Il est évident que ce genre de structure constitue une certaine contrainte stylistique pour le traducteur, mais aussi que celui-ci est tenu d'essayer de la respecter sous peine de faire d'un ouvrage aussi brillant tant par son ton et sa forme que par son contenu un livre scientifique relativement traditionnel. 
Venons-en plus précisément à ce que nous avons appelé les jeux linguistiques dans le titre de cet article. Sous cette appellation assez large, nous avons voulu regrouper un éventail d'astuces formelles allant de purs jeux de mots à des constructions linguistiques très élaborées dans lesquelles fond et forme sont quasiment indissociables.

Pour ce qui est des jeux de mots, c'est-à-dire des purs jeux sur les mots, ils sont légion dans Gödel, Escher, Bach et nous devrons nous contenter d'en citer quelques-uns afin de donner une idée de leur forme et de leur éventuelle fonction.

Ainsi, dans le chapitre IX, qui traite du Zen, on rencontre les termes "zentence » (Zen/sentence) et "zensible" (Zen/sensible). Dans les deux cas, il s'agit de calembours phonétiques, presque homonymiques, à la limite du mot valise. Des astuces aussi locales ont une fonction strictement ludique, et le traducteur n'est certainement pas obligé d'introduire systématiquement un jeu de mots chaque fois qu'il y en a dans l'original. L'important est de reproduire l'effet voulu, lequel peut être déplacé et recréé dans un cadre plus global, comme celui d'un paragraphe, voire d'un chapitre.

Dans les cas cités, il était possible de produire, en français, des calembours construits selon le même principe, ce qui a donné "zensée " (Zen/sensée) et "zentagme " (Zen/syntagme).

Ce principe de la «densité » humoristique d'un texte implique que le traducteur peut parfois négliger un jeu de mots donné de l'original s'il estime que le strict respect de sa position et de sa structure ne pourrait aboutir qu'à un équivalent " tiré par les cheveux »; il peut alors en "créer » un autre qui, ailleurs, s'insérera plus naturellement dans le texte. Ainsi, à la fin d'un long exposé sur un casse-tête de logique formelle dont le but est de trouver la chaîne " $M U$ », nous avons traduit la remarque «Have fun! " par "Et aMUsez-vous bien!", que l'auteur n'aurait certainement pas laissé passer s'il avait écrit son livre en français ! Ce type de jeu de mots, fondé sur une inclusion soulignée par la typographie, nous a paru d'autant plus fondé qu'Hofstadter l'utilise lui-même à plusieurs reprises.

Dans le dernier dialogue, véritable mine de divertissements linguistiques en tous genres, le personnage de l'Auteur déclare, en arrivant (dans l'original) : "Sorry I was so late. I followed the wrong road and wound up very far away", ce que nous avons rendu par : «Je suis désolé d'être en retard, mais je me suis trompé de chemin, j'ai pris la rue du Cherche-Midi à quatorze heures et me suis retrouvé au diable vauvert, non loin de l'avenue d'Humaines. " Tout francophone y reconnaîtra l'idiotisme "chercher midi à quatorze heures » et tout Parisien saisira l'allusion à deux voies de la capitale, la rue du Cherche-Midi et l'avenue du Maine. Certains estimeront peut-être qu'il s'agit la d'une "sur-traduction » gratuite, mais l'essentiel est que la plaisanterie recréée soit dans l'esprit de l'auteur et du livre.

Là encore, en créant de toutes pièces ces deux calembours, nous avons repris des formes de jeux linguistiques qui se retrouvent ailleurs dans l'original. Citons, pour ce qui est des calembours homonymiques sur des noms propres, le cas du Fourmilier, baptisé «Aunt Hillary» (inclusion de " ant hill»). Par ailleurs, le clin d'oeil implicite à la maison d'édition française, sise avenue du Maine, à Paris, se situe dans la même veine que la "signature cachée" de l'auteur dans deux des répliques de la dernière page du livre ("Achilles : (...) a host of formal structural tricks." «Author : After describing the Endlessly Rising Canon (...) "). C'est une première illustration de la fonction cryptologique (codage) de certains jeux linguistiques, fonction que nous retrouverons à de plus hauts niveaux dans d'autres astuces sur les mots et les structures dans Gödel, Escher, Bach.

Un niveau au-dessus de ces calembours, que l'auteur lui-même a parfois marqués " silly pun " dans la marge du texte annoté, on trouve des astuces moins ponctuelles, qui ont un effet moins local et nécessitent une certaine adaptation en amont ou en aval. 
Ainsi, dans le dernier dialogue du livre, intitulé Six-part Ricercar ("Ricercar à six voix "), l'exclamation "The grounds are excellent!" surgit cinq fois de suite dans la bouche des personnages, suivie de quelques phrases faisant comprendre au lecteur que sa signification change chaque fois. Voici, en résumé, ce que l'on a dans l'original :

1. Babbage : The grounds are excellent! We had just enough light to see how well maintained they are (...).

[Ensuite, il parle du parc dans lequel il vient de se promener. Il est donc clair que "grounds" signifie terrain, jardin.]

2. Babbage [parlant avec modestie de la machine à jouer aux échecs qu'il a construite] : (...) $I ' m$ sure that there are very few grounds for praise, in this case.

Crab : The grounds are excellent! All you need to do is look at the board and see for yourself $(. .$.$) .$

[Le Crabe est fait échec et mat par la machine et trouve, contrairement à Babbage, qu'il y a bien des raisons de louer l'habileté de celle-ci.]

3. Tortoise : The grounds are excellent II think that the problem lies instead with the input leads (...).

[Les termes «input leads» indiquent clairement qu'il est question de fils électriques et que "grounds" désigne les fils de mise à la terre.]

4. Achilles: The grounds are excellent! At least, they have a delicious aroma (...). [Il s'agit dans ce cas du café qu'il a préparé.]

5. Achilles : (...) What I find so fascinating about this particular print is that not only the figures but also -

Author : The grounds are excellent ! (..)

[Ici, l'Auteur parle de la gravure Verbum d'Escher. " Grounds " désigne donc les fonds, par opposition aux " figures".]

Pour résoudre cette gageure, il nous fallait trouver un mot (ou une expression) ayant quatre ou cinq sens différents et suffisamment courants pour pouvoir les introduire dans le dialogue sans trop d'acrobaties.

Avant de parvenir à notre solution, "Superbe tour!", nous sommes passés par deux étapes : pour commencer, nous avons tout simplement dressé une liste de mots ayant plusieurs sens, mais il n'était pas facile d'en trouver ayant quatre, ou mieux cinq, sens courants différents. Nous avons alors remarqué, parmi nos " candidats ", les deux termes " tour " (nom masculin) et " tour " (nom féminin) dont les divers sens cumulés suffisaient largement. Il se posait alors un problème tout à fait spécifique au français par rapport à l'original en anglais, puisqu'il nous fallait éluder le genre de ces termes, ce qui a été possible 1) en supprimant tout article et en conservant, comme dans l'original, une expression d'exclamation et 2) en recherchant un ou plusieurs adjectif(s) ayant une forme " neutre", comme " superbe ", qui peut qualifier aussi bien un substantif masculin qu'un substantif féminin.

On peut d'ailleurs remarquer que l'original recourt également à une légère « tricherie " avec l'emploi du pluriel grounds. La polysémie n'aurait en effet pas fonctionné aussi bien au singulier, notamment pour les sens de "raison " et de " café moulu ".

Nous avons donc abouti à la solution "Superbe tour!", dans laquelle le terme " tour " avait les différents sens suivants :

- Le dialogue se déroule dans un château ; le sens de tour/donjon s'insérait donc naturellement ;

- Le Crabe et un programme écrit par Babbage jouent aux échecs. Il était donc facile de placer le mot " tour " dans son sens de pièce du jeu d'échecs; 
- Comme dans l'original, certains personnages pouvaient aller se promener, autrement dit, "faire un tour";

- Certains des personnages étant en train de jouer aux échecs, le « tour " pouvait prendre le sens de coup astucieux ;

- Le sens mécanique de tour d'usinage pouvait faire écho au sens électrique de "grounds" dans l'original.

Cet exemple est une transition entre les jeux de/sur les mots et des jeux plus structuraux en ce que l'astuce de la polysémie nécessite une certaine "préparation du terrain » avant la suite de répliques contenant l'expression "Superbe tour!». Bien entendu, si les remarques préparatoires aux différentes acceptions de " tour " précèdent ce passage du dialogue, nous avons dû d'abord résoudre ce casse-tête, puis remonter dans le texte pour y glisser en amont (comme dans l'original, d'ailleurs) quelques indices préalables, ce qui signifie que nous avons dû procéder à une certaine adaptation de quelques répliques.

Passons à un deuxième cas d'interférence entre le jeu linguistique et son " environnement ». Il se trouve dans le dialogue "Sonata for Unaccompanied Achilles", qui sert à introduire le thème de la différence entre la forme et le fond dans l'art graphique et les mathématiques, développé plus longuement dans le chapitre suivant (chap. III). Ainsi, dans un dessin en noir et blanc, la partie en noir du tableau (la figure) définit totalement son complément, la partie blanche, qui constitue le fond, et vice versa. À travers cette métaphore, $\mathrm{D}$. R. Hofstadter explique un célèbre théorème mathématique qui est aussi un des sujets centraux du livre, le théorème de Gödel.

Le dialogue se présente sous forme d'une conversation téléphonique entre Achille et la Tortue, mais entendue uniquement du côté d'Achille (ce qui ne nous donne seulement le «fond " ou seulement la "figure" de l'échange). La Tortue, qui a mal à la tête ("Achilles: A headache, too? That's a shame. Perhaps you should just go to bed. ") pose une devinette à Achille, qui la répète : " $A$ word with the letters ' $A$ ' ' $D$ ' ' $A$ ' ' $C$ ' consecutively inside it (...) ». Il propose une solution erronée, et pose alors lui aussi une devinette à la Tortue : "What's $a$ word that begins with the letters ' $H E$ ' and also ends with the letters ' $H E$ '? "; la Tortue suggère tout de suite une réponse (qui n'est pas révélée au lecteur) et Achille réplique : "Very ingenious - but that's almost cheating. It's certainly not what I meant ! " On en déduit aisément que la Tortue a trouvé le mot " he ". Elle découvre ensuite le mot auquel pensait vraiment Achille, " headache " (qui n'est cependant pas explicitement révélé dans le dialogue), mais celui-ci ne trouve pas la solution à la devinette de la Tortue et demande un indice. On lit alors : "I don't know what you mean by 'figure' and 'ground' in this case " et l'on se rend compte que les deux personnages s'étaient posés, de deux façons différentes, la même énigme sur le terme " headache ", subtilement introduit au début du dialogue.

Il est évident qu'il était impossible, en français, de conserver " he » et "adac " et qu'il fallait essayer de recréer ce véritable "mot d'esprit ", qui véhicule à lui seul l'idée principale du dialogue et du chapitre suivant. Pour cela, nous devions donc trouver un terme équivalent. Mais que signifie " équivalent » dans un tel cas ? C'est qu'il fallait réunir les conditions suivantes :

a) trouver un terme ayant un début et une fin identiques;

b) que ce début et cette fin (la figure) définissent une unique partie interne (le fond) et vice versa. (En anglais, " headache" est - presque... - le seul terme commençant et finissant par "he»);

c) que le terme choisi soit, autant que possible, assez courant, afin de pouvoir le glisser sans trop de peine dans le dialogue; 
d) que le début et la fin soient, si possible, un terme autonome permettant une solution "dégénérée " à la devinette d'Achille, c'est-à-dire l'équivalent de la «tricherie» de la Tortue avec " he".

Après beaucoup de tâtonnements, nous avons découvert le terme " entretien ", qui satisfaisait toutes les conditions ci-dessus (EN-treti-EN). Cependant là encore, telle une pierre lancée dans une mare et autour de laquelle se forment des ondes concentriques, cette astuce centrale du texte, qu'il nous a fallu trouver avant de pouvoir construire le reste du dialogue autour d'elle, nécessitait une légère adaptation des échanges entre la Tortue et Achille pour y insérer discrètement le terme " entretien ". Il s'agit là d'un dialogue sur la forme et le fond dont le contenu est, en fait, essentiellement exprimé par les devinettes, c'est-à-dire par les jeux linguistiques. Il est clair que dans un tel cas, la "forme» doit être impérativement reproduite.

Le troisième exemple est tiré du "Canon by Intervallic Augmentation * (Canon par augmentation d'intervalles). Ce dialogue précède le chapitre intitule «The Location of Meaning ", dans lequel l'auteur parle longuement des messages et de leurs codages. L'un des thèmes du chapitre est notamment la possibilité, pour un même message, d'avoir plusieurs contenus. D. R. Hofstadter examine plus particulièrement l'existence possible de ce genre de double codage dans l'ADN. L'unité fondamentale du code génétique, le codon, est en effet un triplet de nucléotides (ou des bases que sont l'Adénine, la Guanine, la Cytosine, la Thymine et l'Uracile) identifié par des combinaisons de trois lettres. "AUG ", "UAU ", "CGC " et "CUU " sont quelques exemples de codons et en les enchaînant, on obtient un "message génétique ". " AUGUAUUAACGCCUU " est donc un message génétique qu'on peut décrypter d'au moins deux façons différentes, selon la division en codons choisie. On peut y lire "AUG-UAU-UAA-CGC-CUU » ou, en décalant la grille de découpage d'une unité à droite, "A-UGU-AUU-AAG-CGCUU ". C'est là un mécanisme de double codage effectivement exploité par certains organismes et le sujet illustré par le dialogue.

Le scénario explicite de celui-ci est le suivant : Achille et la Tortue viennent de finir leur repas dans un restaurant chinois et mangent leurs "gâteaux-surprises " (fortune-cookies), lesquels contiennent traditionnellement un petit message. Par inadvertance, Achille avale un bout du sien, et il essaie ensuite, avec l'aide de la Tortue, de le reconstituer. Au début, ils ont quelques difficultés parce qu'il manque tous les espaces entre les lettres. Voici ce message, dans l'original : "ONEWARTWOEAREWE». Achille réussit un premier décodage : "ONE WAR TWO EAR EWE» (remarquez le découpage en mots de trois lettres, essentiel pour l'analogie avec les codons). Il se plaint cependant que cela n'a ni queue ni tête. La Tortue, elle, décode autrement cette séquence de lettres, qu'elle divise différemment. Elle trouve : «O NEW ART WOE ARE $W E$ " (qui pourrait se traduire, littéralement, par "Ô nouvel art, pauvres de nous ") et conclut que "That sounds like an insightful commentary on the new art form of 5/17-haiku».

Toute traduction littérale aurait évidemment détruit ce passage. Or, les termes "one war two ear ewe " ou " o new art woe are we " n'ont, en soi, aucune espèce d'importance. Il fallait que le message créé en français :

- contienne au moins quinze lettres ;

- contienne un nombre de lettres qui soit un multiple de trois;

- permette deux découpages différents, un premier donnant une suite de cinq mots de trois lettres et un deuxième, décalé d'un cran à droite, qui produise une autre suite de mots ayant, cette fois-ci, un sens. 
En fait, la meilleure solution aurait consisté à trouver un message permettant deux décryptages sensés, ce qui aurait été une amélioration par rapport à l'original, dont c'était sans doute l'objectif initial.

Il a été très difficile de résoudre ce problème en français. Pour commencer, il y a beaucoup moins de mots de trois lettres en français qu'en anglais. Ensuite, des trois exigences susmentionnées, seule la première nous laissait une certaine latitude : nous aurions peut-être pu nous contenter d'un message de douze lettres seulement. À l'extrême limite, le nombre de lettres de chaque mot aurait pu être augmenté jusqu'à quatre, mais cela aurait fortement affaibli l'analogie avec les codons, puisque ceux-ci ont une longueur de trois lettres exactement.

Notre résultat final a été : "OILSONTICILESUS ", qui se découpe en " OIL SON TIC ILE SUS ", message tout aussi obscur que "ONE WAR TWO EAR EWE ", et en "O ILS ONT ICI LES US ", dont l'interprétation est qu'il s'agit d'une " réflexion flatteuse » sur le restaurant dans lequel se trouvent Achille et sa compagne.

Pour terminer cette analyse des jeux linguistiques de Gödel, Escher, Bach, nous parlerons d'un des points culminants de ce livre du point de vue du travail sur la forme, le "Crab Canon" (Canon cancrizans). En effet, ce dialogue est structuré comme un $\mathrm{Ca}$ non cancrizans (ou canon à l'écrevisse) en musique. Dans cette forme de canon à deux voix, une voix constitue une mélodie se déroulant « à l'endroit ", ou de " $A$ à $Z$ ", tandis que la deuxième est identique à la première, mais " à l'envers ", c'est-à-dire de " $\mathbf{Z}$ à $\mathbf{A}$ ", ce qui forme donc une structure symétrique. Cette structure, que l'on retrouve en biologie dans l'ADN, rappelle également, par ce jeu de l'inversion, les palindromes, phrases ou expression qui peuvent être lues à l'endroit comme à l'envers (par ex. : " Élu par cette crapule "). La subtilité de ce dialogue, c'est qu'en réalité, il n'y a de véritable palindrome qu'au niveau de la forme du texte. La signification des échanges n'est en effet pas la même dans la première partie du dialogue et dans sa partie symétrique, ce qui implique qu'il a également fallu chercher des expressions polysémiques, ou tout au moins acceptant plusieurs lectures, comme "Ça alors, vous parlez d'un artiste", ou « Il pourrait bien finir au violon".

Interrogeons-nous sur le travail spécifique du traducteur face à un tel casse-tête. Le " fond " du texte est, quasiment en totalité, «sa forme » même, c'est-à-dire le parallélisme 1) avec le canon cancrizans en musique ;2) avec la structure de certains segments d'ADN et 3) avec une gravure d'Escher dans laquelle des rangées de crabes se déplaçant en sens inverse se croisent. Le codage qu'est tout jeu de mots est ici effectué au niveau le plus global, à savoir celui de l'ensemble du texte. Ce qu'il « cache ", c'est aussi qu'il reprend un des grands thèmes du livre, celui de l'autoréférence indirecte (puisque les trois seules répliques "pertinentes" sur les trente-sept du dialogue original - signalées par l'auteur dans ses annotations - décrivent des créations artistiques ou des phénomènes génétiques qui ont la même forme que le dialogue dans lequel ils sont évoqués).

Cette structure est donc, de toute évidence, essentielle au texte puisqu'en son absence, ce dialogue n'aurait plus de raison d'être et les chapitres techniques suivants suffiraient à expliquer l'autoréférence indirecte dans un système formel ainsi que la structure de l'ADN. Exception faite des trois répliques susmentionnées sur Escher, la génétique et Bach, nous avions donc la plus grande liberté en matière de traduction, le problème se réduisant, tout comme dans le cas d'un palindrome, à un pur jeu de virtuosité. Vous pourrez d'ailleurs noter que la version française de ce dialogue est plus courte de quatre répliques. Pour plus de clarté, voici le texte original et notre solution à cette véritable gageure de traduction : 


\section{CRAB CANON}

Achilles and the Tortoise happen upon each other in the park one day while strolling.

Tortoise : Good day, Mr. A.

Achilles : Why, same to you.

Tortoise : So nice to run into you.

Achilles : That echoes my thoughts.

Tortoise : And it's a perfect day for a walk. I think I'll be walking home soon.

Achilles : Oh, really? I guess there's nothing better for you than walking home soon.

Tortoise : Incidentally, you're looking in very fine fettle these days, I must say.

Achilles : Thank you very much.

Tortoise : Not at all. Here, care for one of my cigars?

Achilles : Oh, you are such a philistine. In this area, the Dutch contributions are of markedly inferior taste, don't you think?

Tortoise : I disagree, in this case. But speaking of taste, I finally saw that Crab Canon by your favorite artist, M. C. Escher, in a gallery the other day, and I fully appreciate the beauty and ingenuity with which he made one single theme mesh with itself going both backwards and forwards. But I am afraid I will always feel Bach is superior to Escher.

Achilles : I don't know. But one thing for certain is that I don't worry about arguments of taste. De gustibus non est disputandum.

Tortoise : Tell me, what's like to be your age? Is it true that one has no worries at all?

Achilles : To be precise, one has no frets.

Tortoise: Oh, well, it's all the same to me.

Achilles : Fiddle. It makes a big difference, you know.

Tortoise : Say, don't you play the guitar?

Achilles : That's my good friend. He often plays, the fool. But I myself wouldn't touch a guitar with a ten-foot pole!

(Suddenly, the Crab, appearing from out of nowhere, wanders up excitedly, pointing to a rather prominent black eye.)

Crab : Hallo ! Hulloo! What's up ? What's new? You see this bump, this lump ? Given to me by a grump. Ho ! And on such a fine day. You see, I was just idly loafing about the park when up lumbers this giant fellow from Warsaw - a colossal bear of a man - playing a lute. He was three meters tall, if I'm a day. I mosey on up to the chap, reach skyward and manage to tap him on the knee, saying, "Pardon me, sir, but you are Pole-luting our park with your mazurkas. "But WOW! he had no sense of humor - not a bit, not a wit - and POW! - he lets loose and belts me one, smack in the eye! Were it in my nature, I would crab up a storm, but in the time-honored tradition of my species, I backed off. After all, when we walk forwards, we move backwards. It's in our genes, you know, turning round and round. That reminds me - I've always wondered, "Which came first - the Crab, or the Gene?" That is to say, "Which came last - the Gene, or the Crab ?"I'm always turning things round and round, you know. It's our genes, after all. When we walk backwards, we move forwards. Ah me, oh my ! I must hope along on my merry way - so off I go on such a fine day. Sing " ho l " for the life of a Crab! TATA! Ole!

(And he disappears as suddenly as he arrived.)

Tortoise : That's my good friend. He often plays the fool. But I myself wouldn't touch a tenfoot Pole with a guitar! 
Achilles : Say, don't you play the guitar?

Tortoise : Fiddle. It makes a big difference, you know.

Achilles: Oh, well, it's all the same to me.

Tortoise : To be precise, one has no frets.

Achilles : Tell me, what's it like to be your age? Is it true that one has no worries at all?

Tortoise : I don't know. But one thing for certain is that I don't worry about arguments of taste. Disputandum non est de gustibus.

Achilles : I disagree, in this case. But speaking of taste, I finally heard that Crab Canon by your favorite composer, J.S. Bach, in a concert the other day, and I fully appreciate the beauty and ingenuity with which he made one single theme mesh with itself going both backwards and forwards. But I'm afraid I will always feel Escher is superior to Bach.

Tortoise : Oh, you are such a philistine. In this area, the Dutch contributions are of markedly inferior taste, don't you think?

Achilles : Not at all. Here, care for one of my cigars?

Tortoise : Thank you very much.

Achilles : Incidentally, you're looking in very fine fettle these days, I must say.

Tortoise : Oh really? I guess there's nothing better for you than walking.

Achilles : And it's perfect day for a walk. I think I'll be walking home soon.

Tortoise : That echoes my thoughts.

Achilles : So nice to run into you.

Tortoise : Why, same to you.

Achilles : Good day, Mr. T.

\section{CANON CANCRIZANS}

Achille et la Tortue se rencontrent un jour dans le parc, alors qu'ils se promenaient.

La Tortue : Salut, mon cher Achille!

Achille: Quel plaisir, cette rencontre!

La Tortue : Le hasard nous est favorable, aujourd'hui! J'en suis bien contente.

Achille : $\quad$ Et moi de même.

La Tortue: N'est-ce pas un jour parfait pour se promener? Je vais rentrer chez moi à pied.

Achille : $\quad$ Oui, il n'y a pas mieux que la marche. Et en dépit de nuages, il fait bon, l'air de rien.

La Tortue : De rien... Vous avez vraiment l'air en forme, ces jours-ci.

Achille : Merci beaucoup, vous êtes bien gentille.

La Tortue : Oh non, mais permettez-moi de vous offrir un cigare, par amitié.

Achille: Mon amie, il est clair que vous n'êtes guère connaisseuse en la matière : la contribution hollandaise n'est que de second ordre, question de goût!

La Tortue: Je ne suis pas d'accord! Mais puisque vous parlez de goût, je dois vous dire que j'ai enfin réussi à voir, récemment, le fameux Canon cancrizans de votre artiste favori, M.C. Escher, et j'admets que j'ai vivement admiré l'art, et même le génie, avec lequel il entrelace l'envers et l'endroit d'un même thème. Je n'en continue pas moins à penser que Bach est supérieur à Escher.

Achille : $\quad$ Ça, je ne sais pas. Ce que je sais, par contre, c'est que je ne me soucie pas des affaires de goût. D'ailleurs, les goûts et les couleurs ne se discutent pas !

La Tortue : Parlons plutôt d'autre chose! Je parie que vous ignorez que votre artiste favori a de très solides compétences musicales. Il se pourrait bien qu'il finisse par changer de métier!

Achille: Je sais, mais je me demande s'il changera, un jour.

La Tortue : $\hat{\mathrm{A}}$ mon avis, il pourrait bien finir au violon. 
Achille : Ça alors, vous parlez d'un artiste!

(Tout à coup, surgissant d'on ne sait où, paraît le Crabe, orné d'un énorme bleu à la tempe.)

Le Crabe: Helli, hello! Salut les amis ! Quoi de neuf ? Vous avez vu un peu ce cocard? Bizarre non ? C'est l'œuvre d'une guitare! Et si vous saviez à qui appartenait cet instrument ! Un si beau jour, en plus ! Eh bien voilà : je vagabondais tranquillement dans le parc quand, tout à coup, quoi c'est-y que j'vois ? Une belle jeune esseulée jouant de la guitare sur un banc. Comme c'était doux ! Mon sang n'a fait qu'un demi-tour ! Hop, hop, en cinquante bonds $j$ 'accours et lui susure à l'oreille : "Sacrebleu, Mademoiselle, comme vous grattez bien. Je suis moi-même un musicien hors pair. Si vous avez besoin d'aide, je suis prêt à vous donner un coup de pince ! " $\grave{A}$ ces mots, je ne sais pas pourquoi, elle a sauté sur son banc, bleue de colère, et bing, bang! Quel ouragan! Et quel manque de compréhension! Bien évidemment, j’ai essayé de prendre mes pinces à mon cou en faisant marche arrière, car nous autres, quand nous reculons, nous avançons. C'est dans nos gènes, tournant en rond, y paraît ! ̀̀ vrai dire, je me suis toujours demandé, avant tout : "Qui a précédé l'autre, le Crabe, ou le Gène ? " ou, tourné autrement : "Qui a suivi l'autre, le Gène, ou le Crabe? "Je me pose sans cesse cette question, car après tout, c'est dans nos gènes : quand nous avançons, nous reculons, nous autres. Allez, les amis, le spectacle est terminé ! Rideau ! Salut la compagnie ! Allô ! Olé !

(Et il disparaît plus vite qu'il n'était apparu.)

La Tortue : Ça alors, vous parlez d'un artiste!

Achille : A mon avis, il pourrait bien finir au violon.

La Tortue: Je sais, mais je me demande s'il changera, un jour.

Achille: Parlons plutôt d'autre chose! Je parie que vous ignorez que votre artiste favori a de très solides compétences graphiques. Il se pourrait bien qu'il finisse par changer de métier!

La Tortue : Ça, je ne sais pas. Ce que je sais, par contre, c'est que je ne me soucie pas des affaires de goût. Les goûts et les couleurs ne se discutent pas, d'ailleurs!

Achille : Je ne suis pas d'accord! Mais puisque vous parlez de goût, je dois vous dire que j'ai enfin réussi à entendre, récemment, le fameux Canon cancrizans de votre artiste favori, J.-S. Bach, et j'admets que j'ai vivement admiré l'art, et même le génie, avec lequel il entrelace l'endroit et l'envers d'un même thème. Je n'en continue pas moins à penser qu'Escher est supérieur à Bach.

La Tortue : Mon ami, il est clair que vous n'êtes guère connaisseur en la matière : la contribution hollandaise n'est que de second ordre, question de goût !

Achille : Oh non, mais permettez-moi de vous offrir un cigare, par amitié.

La Tortue : Merci beaucoup, vous êtes bien gentil.

Achille : De rien... Vous avez vraiment l'air en forme, ces jours-ci!

La Tortue : Oui, il n'y a pas mieux que la marche. Et en dépit des nuages, il fait bon, l'air de rien.

Achille: $\quad$ N'est-ce pas un jour parfait pour se promener? Je vais rentrer chez moi à pied.

La Tortue : Et moi de même.

Achille : Le hasard nous est favorable, aujourd'hui! J'en suis bien content.

La Tortue : Quel plaisir, cette rencontre!

Achille : Salut, ma chère Tortue! 
Quelle leçon tirer de ces différents exemples de traduction ou de jeux linguistiques? Que c'est typiquement un domaine où le traducteur doit exercer ses prérogatives et sa sensibilité en supprimant, déplaçant, recréant, etc. Le simple transcodage est le plus souvent impossible et il nous faut, dans chaque cas, évaluer les "pressions "locales et globales afin de déterminer lesquelles entraînent des contraintes incontournables et lesquelles sont secondaires, voire négligeables.

Bien entendu, il ne s'agit pas seulement de "décortiquer " froidement les astuces de l'original ; il faut aussi laisser s'exprimer son humour. On ne peut d'ailleurs certainement aborder une telle traduction que si l'on apprécie l'humour de l'original et si l'on se sent plus ou moins en résonance avec lui. Notre devise, tout au long de ce travail, a été "Traduire, c'est aussi s'amuser un peu ».

Enfin, en conclusion, nous dirons qu'il ne faut pas avoir froid aux yeux et que ce genre de défi, comme ceux qu'ont relevés les traducteurs de Lewis Carroll ou de James Joyce, semble faire reculer les limites de l" intraduisible ", gouffre dans lequel certains éditeurs francophones avaient d'emblée jeté Gödel, Escher, Bach.

Note

- Précisons qu'il a été traduit ou est en cours de traduction en italien, allemand, néerlandais, français, espagnol, japonais, portugais, suédois et chinois... 